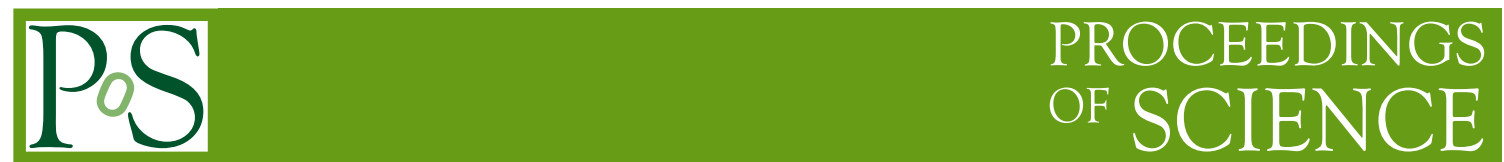

\title{
Revealing proton structure with neural networks
}

\section{Krešimir Kumerički ${ }^{a, *}$}

${ }^{a}$ Department of Physics, Faculty of Science, University of Zagreb, 10000 Zagreb, Croatia

E-mail: kkumer@phy.hr

We report on recent progress in using neural networks to extract information about partonic structure of proton from deeply virtual Compton scattering data.

40th International Conference on High Energy physics - ICHEP2020

July 28 - August 6, 2020

Prague, Czech Republic (virtual meeting)

\footnotetext{
${ }^{*}$ Speaker
} 


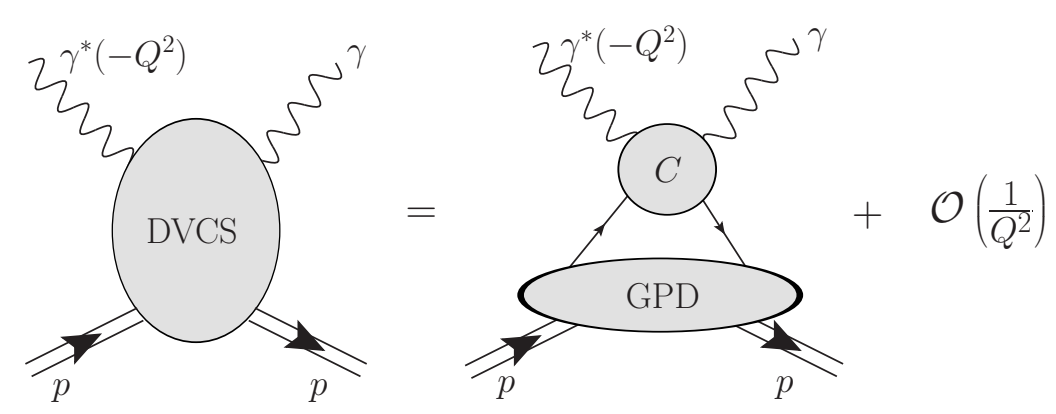

Figure 1: Factorization of DVCS amplitude into convolution of perturbative coefficient function $C$ and GPD.

\section{Introduction}

After decades of measurement and analysis, we have a pretty good understanding of a distribution of quarks and gluons in a fast moving nucleon in terms of their longitudinal momentum, over a large kinematic region. Nevertheless, the full three-dimensional description, including transversal degrees of freedom, is still largely incomplete and is in focus of several experimental facilities, including the future electron-ion collider [1]. Such a description is often encoded in terms of generalized parton distributions (GPDs) [2-4], which are in principle extractable from several hard exclusive processes, with deeply virtual Compton scattering (DVCS) being the most studied (see Refs. [5] and [6] for review of experimental and phenomenological status). In DVCS, a photon with large virtuality $Q^{2}$ scatters off a proton, $\gamma^{*} p \rightarrow \gamma p$, and corresponding quantum amplitude at leading order in $1 / Q^{2}$ factorizes in a convolution of perturbatively calculable coefficient function and a soft GPD function, see Figure 1.

Knowledge of GPD functions would give answers to several outstanding problems, including aforementioned three-dimensional "tomographical" picture of partonic distributions [7], as well as separation of quark contribution to proton spin into orbital and quark spin components [8]. At present, we still don't have good enough knowledge of GPDs to tackle these problems. We are in the intermediate stage, trying to map the Compton form factors (CFFs) describing the DVCS amplitude, i. e., the blob on the left hand side of equation in Figure 1. This is similar to studies of deep inelastic scattering, where extraction of structure functions preceded the extraction of parton distribution functions (PDFs).

Here we report on two studies, where advances in extraction of CFFs enabled some steps forward in understanding the proton structure.

\section{Quark pressure in the nucleon}

One of the fundamental properties of CFFs is given by the dispersion relation, which for the leading-twist $\mathrm{CFF} \mathcal{H}$ takes the form [9]

$$
\mathfrak{R e} \mathcal{H}(\xi, t)=\Delta(t)+\frac{1}{\pi} \mathrm{P} . \mathrm{V} \cdot \int_{0}^{1} \mathrm{~d} x\left(\frac{1}{\xi-x}-\frac{1}{\xi+x}\right) \mathfrak{I m} \mathcal{H}(x, t),
$$

where $\xi=x_{\mathrm{B}} /\left(2-x_{\mathrm{B}}\right), x_{\mathrm{B}}$ is the Bjorken scaling variable, and $t$ is the squared momentum transfer to the nucleon. $\Delta(t)$ is a subtraction constant which can be related to the so-called D-term [10] and, 

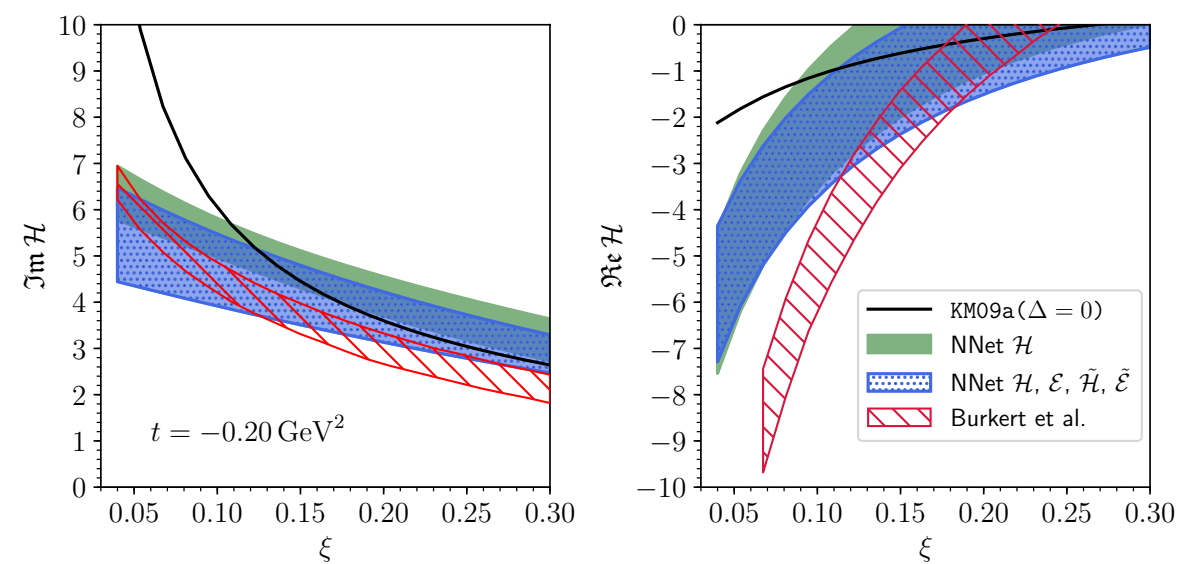

Figure 2: Imaginary (left) and real (right) part of $\mathcal{H}(\xi, t)$ resulting from fits by Burkert et al.[12] and by neural net (NNet) method to the CLAS DVCS beam-spin asymmetry[15] and cross-section[16] data. Blue band corresponds to fit of all four leading CFFs, while green band corresponds to fit of only $\mathcal{H}$. Fit from Ref. [13] with zero subtraction constant is also shown for comparison.

in turn, to the quark pressure [11]. Such analysis and extraction of information about pressure from DVCS scattering data was attempted in Ref. [12], using the model proposed in Ref. [13]. To assess the systematic uncertainty due to the choice of the model we repeated the analysis, using the similar approach and the same DVCS dataset, but parametrizing the CFFs using neural networks. Resulting $\mathfrak{I m} \mathcal{H}$, which dominates the relevant DVCS amplitude, turned out almost the same, see the left panel on Figure 2, but the subdominant $\mathfrak{R e} \mathcal{H}$, see the right panel on Figure 2, has significantly larger uncertainties than in Ref. [12], and different mean values, and corresponding subtraction constant and, in turn, pressure, turns out to be consistent with zero [14]. Still, the method works in principle and with plenty of precise data coming from the JLab $12 \mathrm{GeV}$ facility in the following years there is a clear hope for unambigous determination of quark pressure.

\section{Separation of quark flavors}

In second application of this framework [17] we again use the dispersion relations to constrain CFFs. Constraints are implemented by using neural networks to parametrize, besides imaginary parts of four leading CFFs, only the single subtraction constant $\Delta(t)$, see Figure 3. Real parts of CFFs are then obtained using Eq. (1). This model was then fitted to the complete set of JLab proton DVCS data (see Ref. [17] for details) and it was possible to extract the six leading CFFs, as displayed on Figure 4.

Then, we added the data from the recent measurement of DVCS off neutron [18], and attempted to fit the same neural network model, but now with separate parametrization of up and down quark parts, using the isospin symmetry to relate neutron GPDs to proton ones. This resulted in a clear separation of up and down quark contributions to the leading CFF $\mathcal{H}$, as shown on right panels of Figure 5. Similar separation was attempted by the authors of [18], using local fitting in each kinematic bin, but extracted flavored CFFs were not so cleanly separated in this work. This illustrates both the usefulness of the neural network approach and power of dispersion relations. 


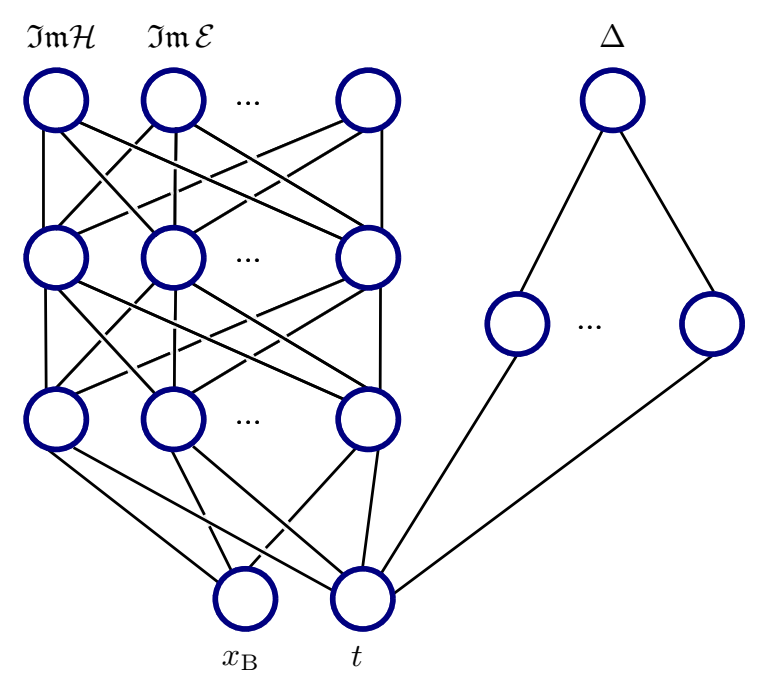

Figure 3: Neural net architecture.
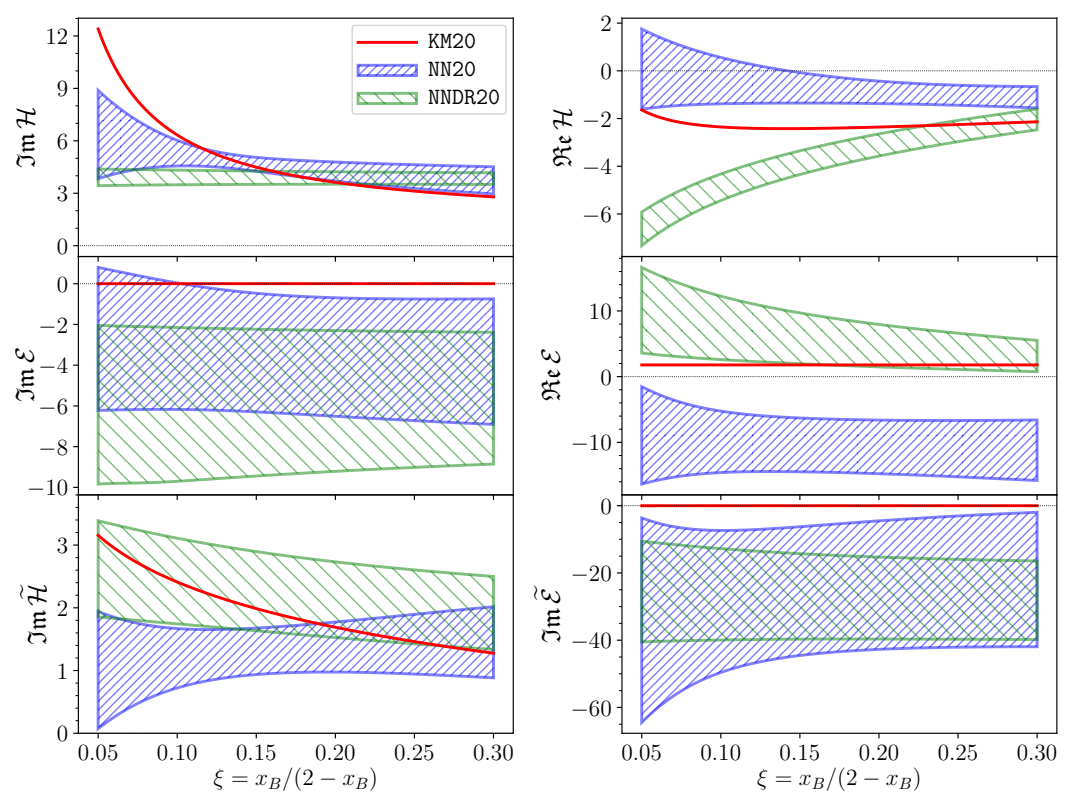

Figure 4: CFFs as extracted at $Q^{2}=4 \mathrm{GeV}^{2}$ and $t=-0.2 \mathrm{GeV}^{2}$ by fits to JLab proton DVCS data. KM20 is fit to parametrization from [13], NN20 is standard simple neural network parametrization, while NNDR20 additionally includes DR constraints.

\section{Acknowledgments}

This work is supported by the Croatian Science Foundation project IP-2019-04-9709, by Deutsche Forschungsgemeinschaft (DFG) through the Research Unit FOR 2926, "Next Generation pQCD for Hadron Structure: Preparing for the EIC", project number 40824754, by QuantiXLie Centre of Excellence through grant KK.01.1.1.01.0004, and the EU Horizon 2020 research and innovation programme, STRONG-2020 project, under grant agreement No 824093. 


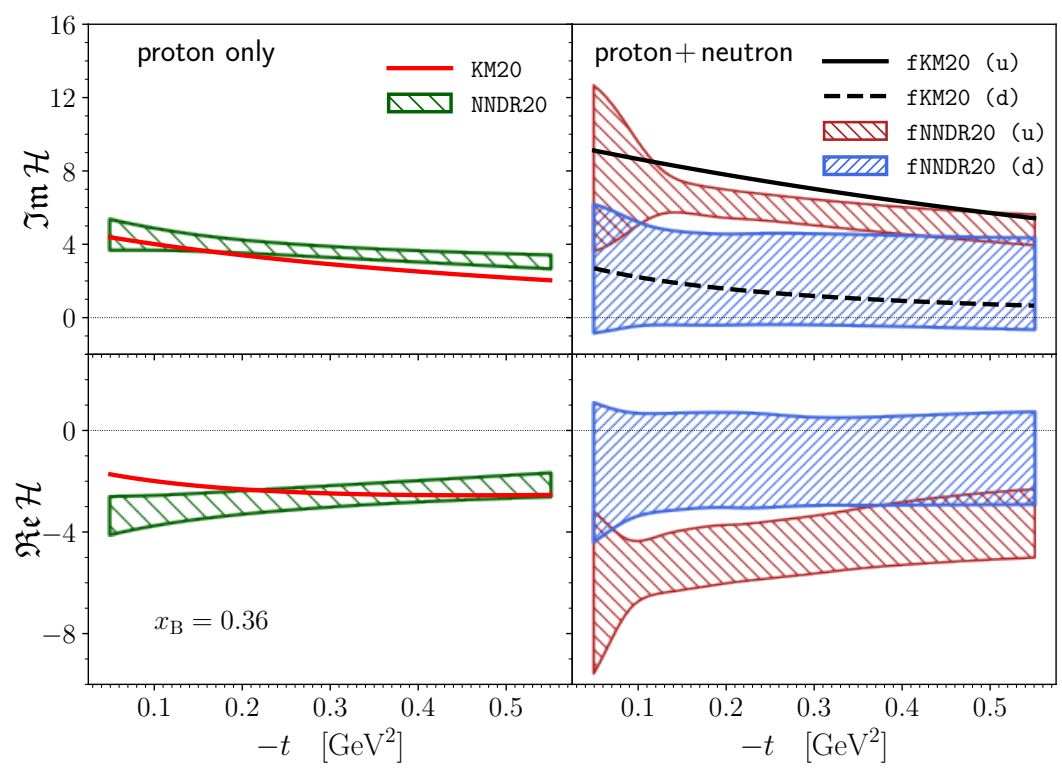

Figure 5: CFF $\mathcal{H}$ extracted (at $Q^{2}=4 \mathrm{GeV}^{2}$ and $x_{\mathrm{B}}=0.36$ ) from neural network fit to proton-only DVCS data (left). Separation of $u$ (red band) and $d$ (blue band) quark CFF $\mathcal{H}$ resulting from neural network fit to proton and neutron JLab DVCS data (right), where $\mathcal{H}=\left(4 \mathcal{H}_{u}+\mathcal{H}_{d}\right) / 9$. Red solid (unflavored), black solid $(u)$ and dashed $(d)$ lines correspond to analogous least-squares model fit to the same data.

\section{References}

[1] A. Accardi et. al., Electron Ion Collider: The Next QCD Frontier, Eur. Phys. J. A52 (2016), no. 9268 [1212.1701].

[2] D. Müller, D. Robaschik, B. Geyer, F. M. Dittes and J. Hořejší, Wave functions, evolution equations and evolution kernels from light-ray operators of QCD, Fortschr. Phys. 42 (1994) 101 [hep-ph/9812448].

[3] A. V. Radyushkin, Scaling limit of deeply virtual Compton scattering, Phys. Lett. B380 (1996) 417-425 [hep-ph/9604317].

[4] X.-D. Ji, Deeply-virtual compton scattering, Phys. Rev. D55 (1997) 7114-7125 [hep-ph/9609381].

[5] N. d'Hose, S. Niccolai and A. Rostomyan, Experimental overview of Deeply Virtual Compton Scattering, Eur. Phys. J. A52 (2016), no. 6151.

[6] K. Kumerički, S. Liuti and H. Moutarde, GPD phenomenology and DVCS fitting, Eur. Phys. J. A52 (2016), no. 6157 [1602.02763].

[7] M. Burkardt, Impact parameter dependent parton distributions and off-forward parton distributions for zeta $\rightarrow$ O, Phys. Rev. D62 (2000) 071503 [hep-ph/0005108]. Erratum-ibid.D66:119903,2002.

[8] X.-D. Ji, Gauge invariant decomposition of nucleon spin, Phys. Rev. Lett. 78 (1997) 610-613 [hep-ph/9603249]. 
[9] O. V. Teryaev, Analytic properties of hard exclusive amplitudes, hep-ph/0510031.

[10] M. V. Polyakov and C. Weiss, Skewed and double distributions in pion and nucleon, Phys. Rev. D60 (1999) 114017 [hep-ph/9902451].

[11] M. V. Polyakov, Generalized parton distributions and strong forces inside nucleons and nuclei, Phys. Lett. $\mathbf{B 5 5 5}$ (2003) 57-62 [hep-ph/0210165].

[12] V. D. Burkert, L. Elouadrhiri and F. X. Girod, The pressure distribution inside the proton, Nature 557 (2018), no. 7705 396-399.

[13] K. Kumerički and D. Müller, Deeply virtual Compton scattering at small xB and the access to the GPD H, Nucl. Phys. $\mathbf{B 8 4 1}$ (2010) 1-58 [0904 . 0458].

[14] K. Kumerički, Measurability of pressure inside the proton, Nature 570 (2019), no. 7759 E1-E2.

[15] CLAS Collaboration, F. X. Girod et. al., Deeply Virtual Compton Scattering Beam-Spin Asymmetries, Phys. Rev. Lett. 100 (2008) 162002 [0711.4805].

[16] CLAS Collaboration, H. Jo et. al., Cross sections for the exclusive photon electroproduction on the proton and Generalized Parton Distributions, Phys. Rev. Lett. 115 (2015), no. 21 212003 [1504.02009].

[17] M. Čuić, K. Kumerički and A. Schäfer, Separation of Quark Flavors using Deeply Virtual Compton Scattering Data, Phys. Rev. Lett. 125 (2020), no. 23232005 [2007. 00029].

[18] M. Benali et. al., Deeply virtual Compton scattering off the neutron, Nature Phys. 16 (2020), no. 2 191-198. 